\title{
Animal Trial
}

\section{e Comparative Study of Chronic Postischemic Pain Models in Mice: O-Ring Versus Tie Method}

Yong Han Seo, MD', Ye Won Gil, MD', Gyuho Choe, MD, $\mathrm{PhD}^{2}$, Sung Hyun Lee, MD, $\mathrm{PhD}^{3}$, Sie Hyeon Yoo, MD, PhD' ${ }^{1}$, and Hue Jung Park, MD, PhD ${ }^{2}$

From: 1Soonchunhyang University Hospital Cheonan; 2 Catholic University of Korea School of Medicine; 3 Kangbuk Samsung Medical Center

Address Correspondence: Sie Hyeon Yoo, Md, PhD Soonchunhyang University Hospital Cheonan 31, Suncheonhyang 6-gil, Dongnam-gu, Cheonan-si, Chungcheongnam-do, Republic of Korea E-mail:

yshyun@schmc.ac.kr

Disclaimer: This research was supported by Basic Science Research Program through the National Research Foundation of Korea (NRF) funded by the Ministry of Education (grant number. 2017R1D1A1B03028360). Conflict of interest: Each author certifies that he or she, or a member of his or her immediate family, has no commercial association (i.e., consultancies, stock ownership, equity interest, patent/licensing arrangements, etc.) that might pose a conflict of interest in connection with the submitted manuscript.

Manuscript received: 03-06-2019 Revised manuscript received: 05-29-2019

Accepted for publication: 07-23-2019

Free full manuscript: www.painphysicianjournal.com
Background: The success rate for the production of animal models of chronic postischemia pain (CPIP) using an O-ring has yet to be improved in the study of complex regional pain syndrome-type I (CRPS-I), and producing a CPIP model is challenging, especially for mice.

Objectives: We devised a new CPIP model with a higher success rate that induces ischemia for 3 hours by tying the hind limbs of mice with a rubber band, followed by reperfusion.

Study Design: A randomized, controlled animal trial.

Methods: Twenty-two male C57BL/6 mice were divided into a sham $(n=6)$, a ring $(n=8)$, and a tie group $(n=8)$. Anesthesia was induced using isoflurane. A precut O-ring was mounted on the upper left ankle in the sham group. A tight-fitting O-ring and a push-pull gauge manometer were mounted at the same location in the ring and tie groups, respectively. Reperfusion was induced 3 hours later. The thickness and circumference of the hind paws were measured before ischemia induction. Measurements were repeated 10 days after reperfusion. Mechanical allodynia was measured with a von Frey filament until 12 weeks after reperfusion.

Results: The new tie model required 5 additional days until the onset of allodynia compared with the existing CPIP O-ring model. However, the successful induction rate of CPIP was higher in the tie group than in the ring group, and allodynia was maintained for over 30 days in the tie group. The ring and tie groups exhibited significantly high levels of tumor necrosis factor-alpha than those in the sham group.

Limitations: First, we did not evaluate hyperalgesia, cold or heat allodynia. Second, we did not measure blood levels of inflammatory or antiinflammatory cytokines, and research on oxidative stress biomarkers such as isoprostane, 8-hydroxy-2'-deoxyguanosine (a marker of DNA oxidative damage), and malondialdehyde was not performed.

Conclusions: The new CPIP tie model has a higher rate of successful induction than existing O-ring models for mice, with longer duration of mechanical allodynia. The model may reduce the number of animals sacrificed in CRPS-I research and could be useful for studying long-term effects of drugs.

Key words: CPIP, mouse, O-ring, rubber band, reperfusion, allodynia, hyperalgesia

Pain Physician 2020: 23:E51-E60 omplex regional pain syndrome (CRPS) is characterized by local, continuous, spontaneous levoked pain that does not coincide with the progress or level of pain due to trauma or a lesion that is typically expected (1). Pain characteristics of CRPS may be not transmitted through specific neural regions or dermatomes but occurs locally and exhibits various patterns of progression including paresthesia, abnormal 


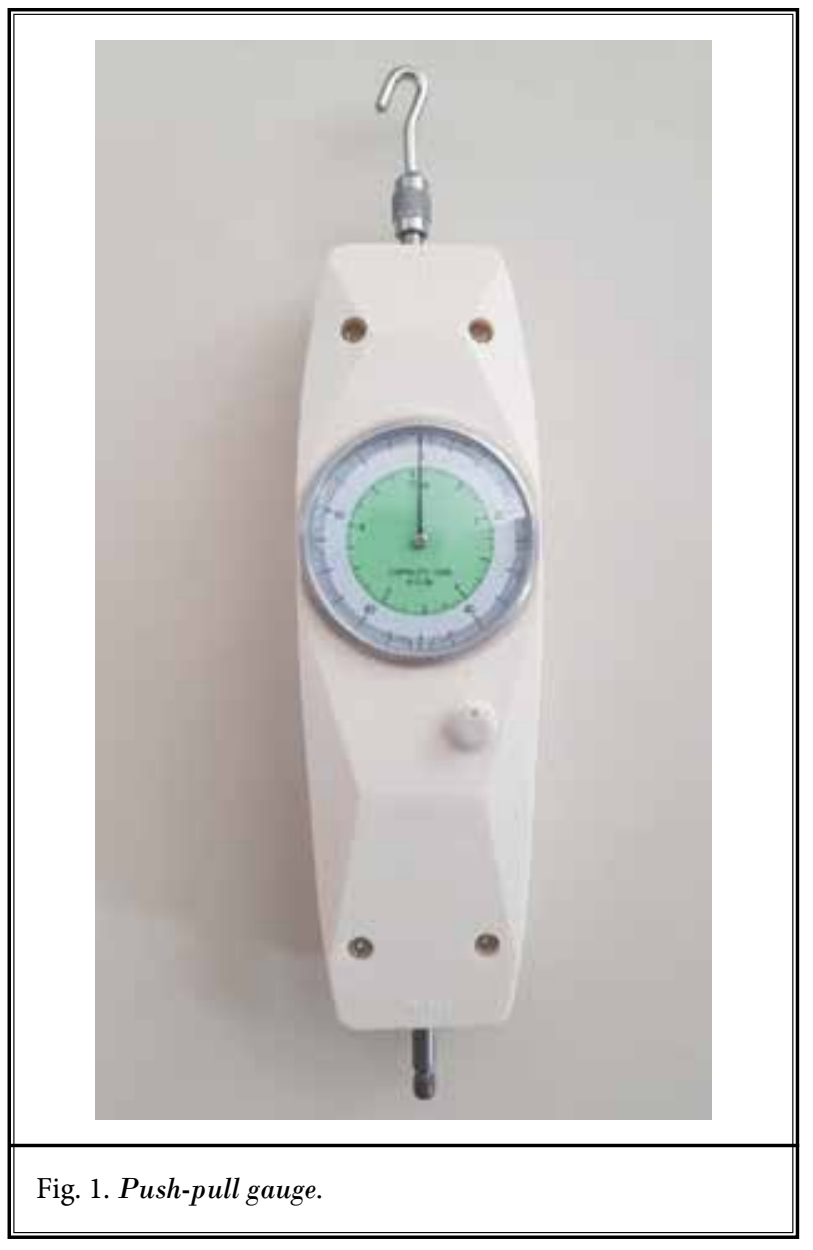

vasomotor responses, motion impairment, abnormal sweating, and dystrophic changes. CRPS is generally divided into 2 types: CRPS type I (CRPS-I), which is characterized by the absence of definable neurologic damage, and CRPS type II, which is accompanied by neurologic damage. Despite extensive research, the mechanism underlying CRPS remains unclear, and the diagnosis and treatment remain challenging.

Since its introduction in 2004, the chronic postischemia pain (CPIP) rat model using an O-ring has been employed in various studies on CRPS-I (2). This model mimics the essential features of CRPS- 1 in humans very well, allowing researchers to study the mechanism of CRPS. However, the rate of successful model production varies among researchers, resulting in an increased number of sacrificed experimental animals, and the overall research period may be longer due to repeated model production. Furthermore, because allodynia lasts approximately 1 month (2), it is difficult to monitor the continuous effect of a drug over a longer period. In addition, it is difficult to apply an O-ring of appropriate size in mice, which are smaller than rats, and there have been few studies using a CPIP-model with an O-ring in mice (3). No research has been reported on CPIP model production itself in mice.

We present a new method that can increase the rate of successful CPIP model induction and can maintain mechanical allodynia for 50 days or more by tying the hind limb of a mouse with a rubber band and using a push-pull gauge with uniform force.

\section{Methods}

\section{Animals}

This study was approved by the Institutional Animal Care and Use Committee of the Catholic University College of Medicine (No: CUMC-2017-0158-01). Male C57BL/6 mice $(20-25 \mathrm{~g})$ were used in the experiment, they were allowed to freely drink and eat, and a 12/12 hour light/dark cycle was used. All animals had 7 days to acclimatize to their environment.

\section{Production of the CPIP Model}

Twenty-two male mice were randomly assigned to either the sham $(n=6)$, ring $(n=8)$ or tie group ( $n$ $=8$ ). General anesthesia was induced using $1.5 \%$ isoflurane and $100 \% \mathrm{O}_{2}$ in all groups. In the ring group, the method used by Coderre et al (2) was used, O-ring with a 5/64 inch internal diameter (AS568-004) that matched the size of the mouse hind limb was placed on the upper part of the left ankle (immediately above the medial malleolus) for 3 hours. In the sham group, precut O-rings of the same size were used to prevent a tightening force. In the tie group, a rubber band (cross section $1 \times 1.5 \mathrm{~mm}^{2}$, elasticity coefficient $0.588 \mathrm{kgf} / \mathrm{cm}^{2}$, Dong In Co., Vung Tau, Vietnam) was cut into a linear shape. One tip of the rubber band was connected to the push-pull gauge (NK-100N, TRIPOD, Wenzhou, China; Fig. 1), and a knot was created at the upper part of the left ankle. A 300-g load was applied to pull the rubber band and create a uniform tightening force (Fig. 2). The rubber band was then wrapped around the limb and tied once again on the opposite side of the ankle, to create a second knot with the same amount of force. In all groups, the tied O-ring and rubber band were removed after 3 hours of ischemia to induce reperfusion, and the mice were awakened from the anesthesia. 


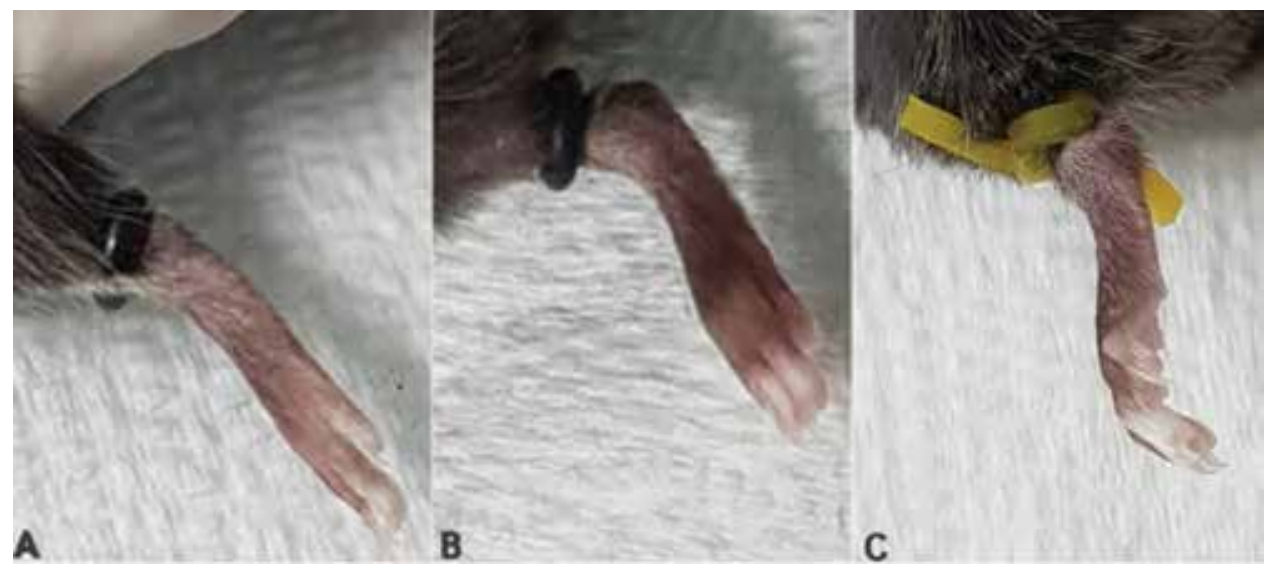

Fig. 2. Ischemic method by group. A. Pre-cut O-ring (sham group; B. O-ring (Ring group); C. Tied with rubber band (Tie group).

\section{Evaluation of Postreperfusion Edema}

The thickness and circumference of the left hind paw were measured before the experiment and at 10 minutes, 1 hour, and 1, 3, 5, 7, and 10 days after reperfusion to observe the progress of edema in each group. To minimize mouse movement, general anesthesia was induced using isoflurane. A micro digital caliper (BD500-100, Bluebird, Shanghai, China) was used to measure the thickness of the left hind paw. To measure the circumference, a string was looped around the left hind paw, and the length of the string was measured with a micro digital caliper.

\section{Evaluation of Mechanical Allodynia}

Mechanical allodynia was measured every 2 days from preexperiment to 85 days after reperfusion. To stimulate the plantar surface, a mouse was placed on a wire mesh floor, and a transparent $8 \times 8 \times 18-\mathrm{cm}$ plastic box was placed over the mouse. After the mouse had acclimatized to the environment for approximately 30 minutes, a force was vertically applied to the mouse's paw for 3 seconds with a von Frey filament (18011 Semmes-Weinstein filament, Stoelting Co., Wood Dale, IL), such that the filament bent in the midplantar area, and the avoidance response was then evaluated. Seven filaments were used, with weights ranging from 2.44 to $4.31 \mathrm{~g}$. The simplified up-down method used by Bonin et al (4) was used to examine the reflex 4 additional times, beginning when the mouse began to show or discontinued showing an avoidance response. A 50\% response threshold was measured based on the reflex patterns and log-value of the von Frey filament.

\section{Histology}

For the histologic aspect, we produced separately 30 new mice and randomized them into 3 groups (sham group, $\mathrm{n}=6$; ring group, $\mathrm{n}=12$; tie group, $\mathrm{n}=$ 12 ) in the way mentioned earlier. At 14 days postreperfusion, a total of 16 mice (sham group, $n=4$; ring group, $n=6$; tie group, $n=6$ ) with paw withdrawal thresholds showed 0.6 or less were deeply anesthetized with ketamine and perfused with $0.9 \%$ saline solution, followed by $4 \%$ paraformaldehyde in $0.1 \mathrm{M}$ phosphate buffer via the cardiovascular system. The ipsilateral L3-L5 spinal cord tissues were collected and postfixed in $4 \%$ paraformaldehyde, then placed overnight in $25 \%$ sucrose at $4^{\circ} \mathrm{C}$. Spinal cord tissues were sliced transversely into $10 \mu \mathrm{m}$ sections on a cryostat (Microm HM 525, Thermo Fisher Scientific, Walldorf, Germany). The sections were blocked with $10 \%$ normal goat serum in phosphate-buffered saline (PBS) containing $0.1 \%$ Triton X-100 (PBST) (Thermo Fisher Scientific, Walldorf, Germany) at room temperature for 2 hours, then incubated with rabbit antimouse tumor necrosis factor-alpha (TNF- $\alpha$ ) polyclonal antibodies (1:100; BA14901; Wuhan Boster Bio-Engineering Co., Ltd., Wuhan, China) at $4^{\circ} \mathrm{C}$ overnight. After they had been washed in PBS (Maixin Biological Technology Development Co., Fujian, China), the TNF- $\alpha$ binding sites were visualized with antirabbit immunoglobulin antibodies 
conjugated with Alexa-594 (1:1000, Molecular Probes, Eugene, OR). Nuclei were counterstained with Topro3 (1:500; Invitrogen , Thermo Fisher Scientific, Walldorf, Germany). At least 3 images were obtained for each mouse using confocal microscopy (Zeiss LSM/510 Upright 2 photon; Carl Zeiss, Jena, Germany) and stored on digital media.

The relative fluorescence intensity of the immunofluorescent images were calculated by Image J software version 1.52a (National Institutes of Health, Bethesda, MD). TNF- $\alpha$ staining was quantified by measuring the total integrated intensity of pixels divided by the total number of pixels in a standardized area. The staining intensity was examined in laminae I and II of the superficial dorsal horn by using a standardized box with 4 to 6 mice per group. Only pixels above a preset background threshold were included. TNF- $\alpha$ data were presented as the total threshold intensity area. Statistics were performed on raw data values.

\section{Statistical Analysis}

All data were expressed as the mean \pm standard error of the mean. Statistical analysis was performed by using GraphPad Prism 7 (GraphPad Software, La Jolla, CA).

A 2-way repeated-measures analysis of variance was used to compare the difference in the $50 \%$ von Frey threshold over time, and the sole thickness and circumference. The Bonferroni multiple comparison test was used for post hoc analysis. The level of statistical significance was set at $P<0.05$.

\section{ResUlts}

There was no significant difference in the weight gain between the 3 groups during the entire research period (Fig. 3).

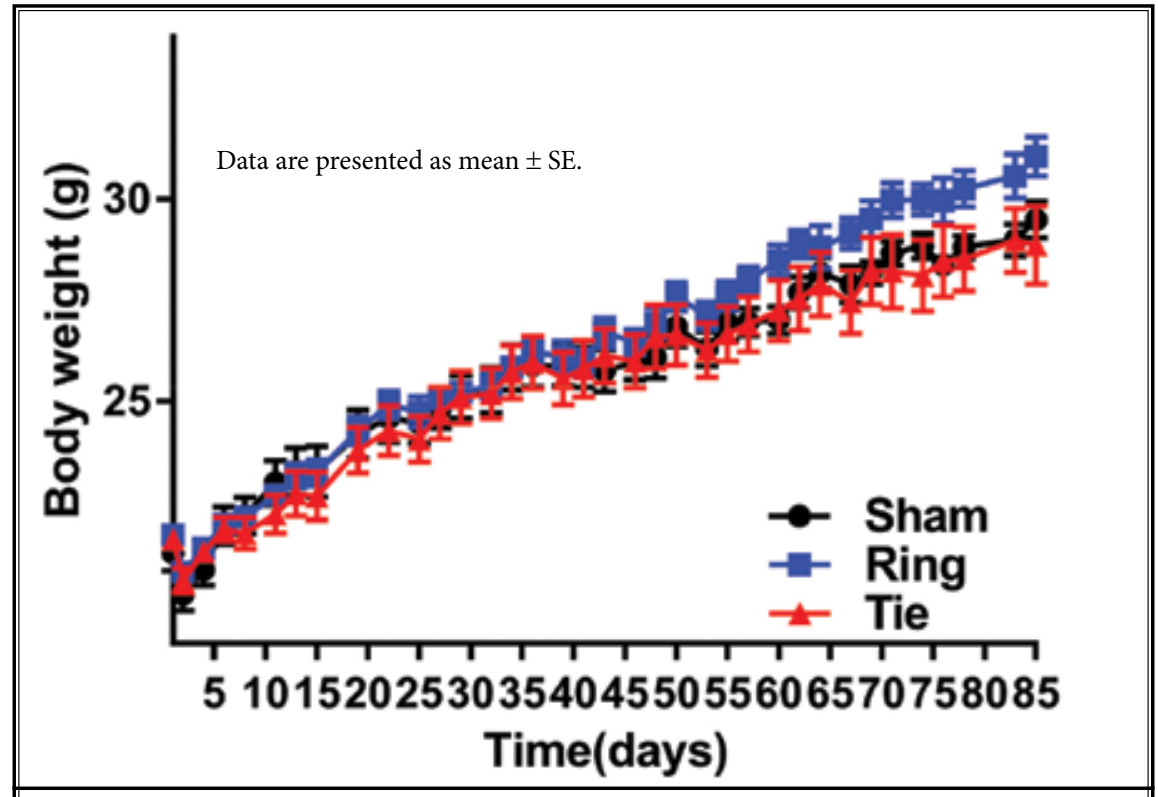

Fig. 3. Body weights over time in each group.

\section{Postreperfusion Hyperemia and Edema}

The ring group showed a more rapid return to a hyperemic state following reperfusion compared with the tie group (Figs. 4 and 5). The ring group showed postreperfusion edema at 10 minutes after reperfusion, then returned to normal at 1 day after reperfusion. In contrast, postreperfusion edema occurred 1 hour after reperfusion in the tie group, the edema extent was greater than in the other 2 groups at 1 day after reperfusion. Edema in the tie group was significantly greater than in the sham group until 3 days after reperfusion and began to return to normal at 5 days after reperfusion (Fig. 5).

\section{Mechanical Allodynia}

The left paw withdrawal threshold significantly decreased at 8 days and 13 days after reperfusion in the ring and tie groups, respectively, compared with the sham group. The mean duration of allodynia were 22 days in the ring group and 55 days in the tie group. There was no difference in the paw withdrawal threshold between the ring and sham groups beginning at 25 days after reperfusion, the tie group had a longer duration of allodynia than the ring group between 30 and 55 days after reperfusion (Fig. 6).

Allodynia sporadically occurred in the contralateral hind paw at 22 and 50 days. This occurrence was significantly greater in the tie group than in the sham group (Fig. 7).

The number of mice with paw withdrawal thresholds of 0.6 or less was higher in the tie group than in the ring group at 

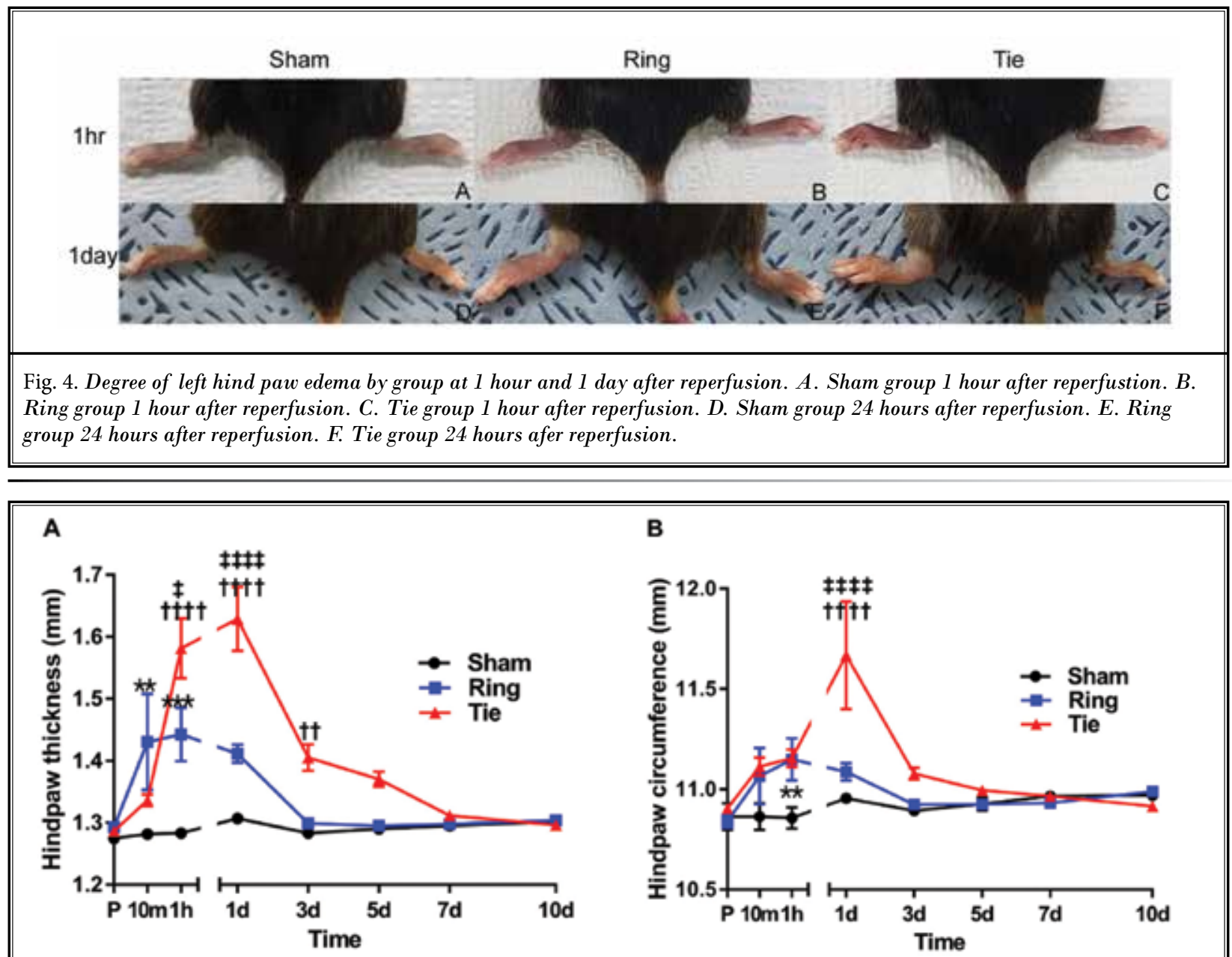

Fig. 5. Thickness and circumference of left hind paw over time.

Measurements of (a) thickness and (b) circumference of left hind paw over time in each group. Data are presented as mean \pm $S E$. (a) Ring group compared with Sham group ${ }^{* *} P<0.01,{ }^{* * *} P<0.001$. Tie group compared with Sham group $\dagger \dagger P<0.01$, $+\dagger+P<0.0001$. Tie group compared with ring group $\neq P<0.05, \hbar \hbar \neq P<0.0001$. (b) Ring group compared with Sham group ${ }^{*} P<0.01$, Tie group compared with Sham group $\dagger \dagger \dagger \dagger P<0.0001$. Tie group compared with Ring group $\not \neq \neq P<0.0001$.

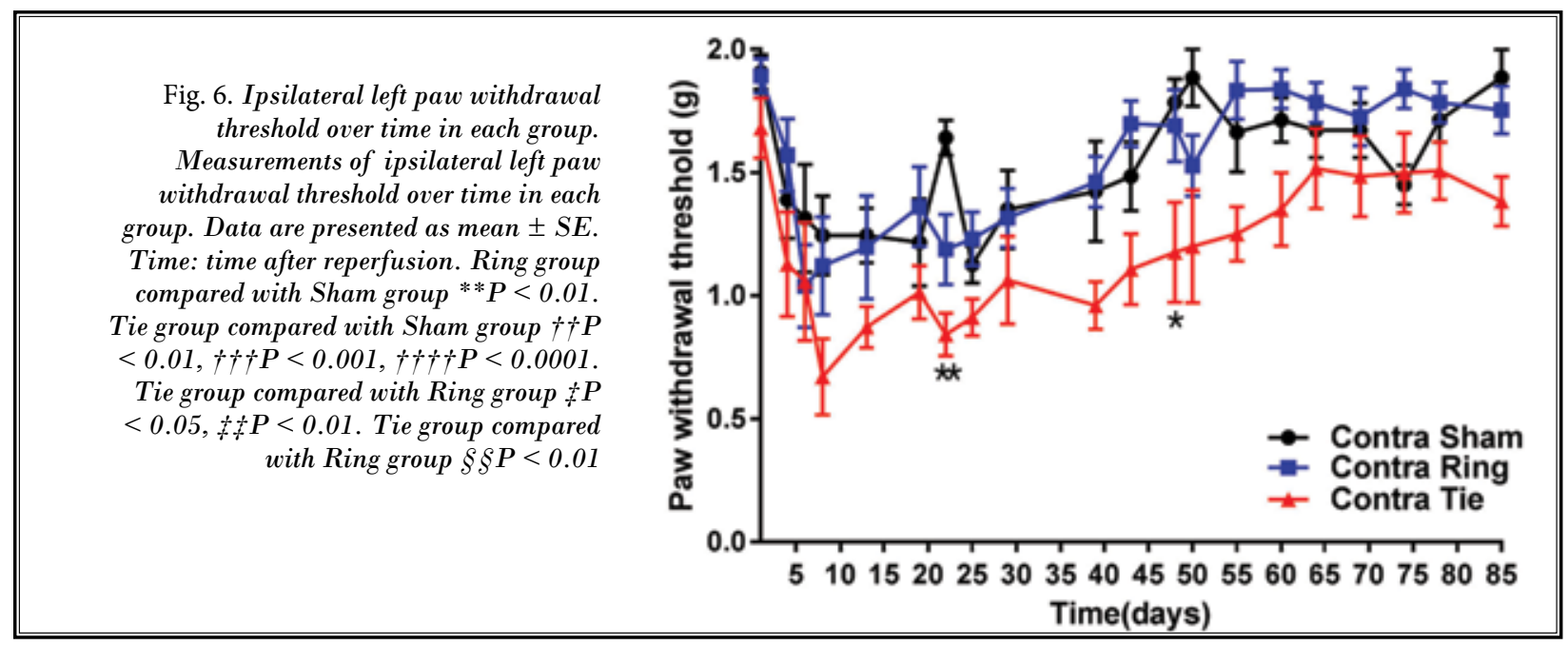




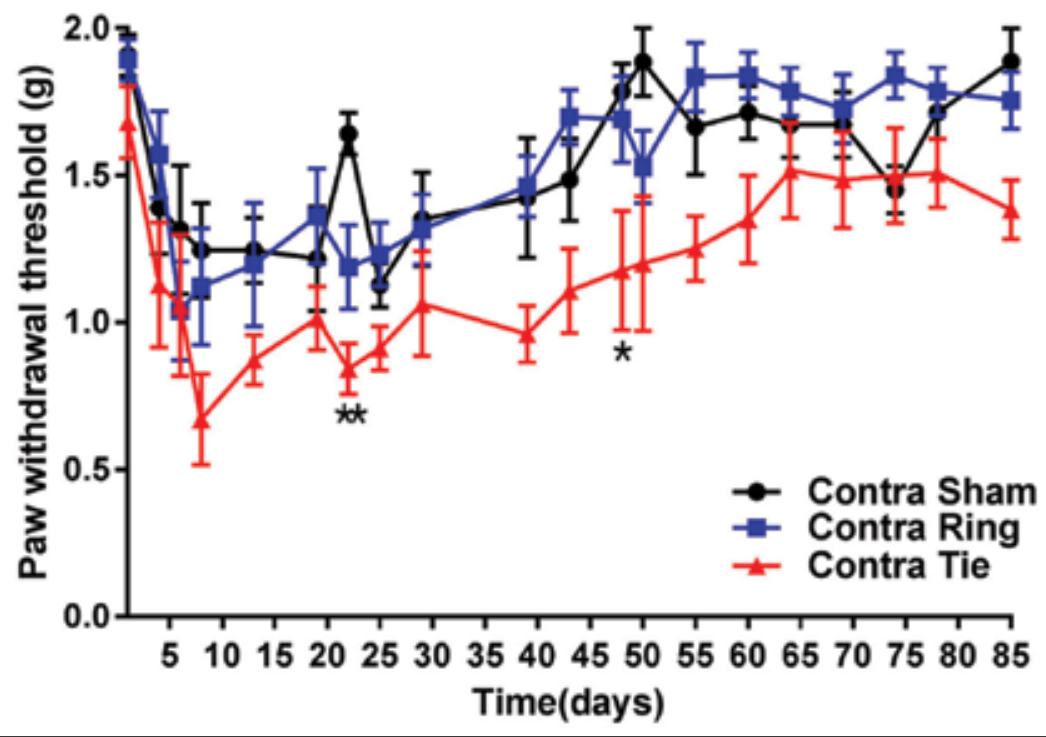

Fig. 7. Contralateral right paw withdrawal threshold over time for each group.

Data are presented as mean

\pm SE. Contra Sham: paw withdrawal threshold for the right hind paw in Sham group; Contra Ring: paw withdrawal threshold for the right hind paw in Ring group; Contra Tie: paw withdrawal threshold for the right hind paw in Tie group. Tie group compared with Sham group ${ }^{*} P<0.05, * * P<0.01$.

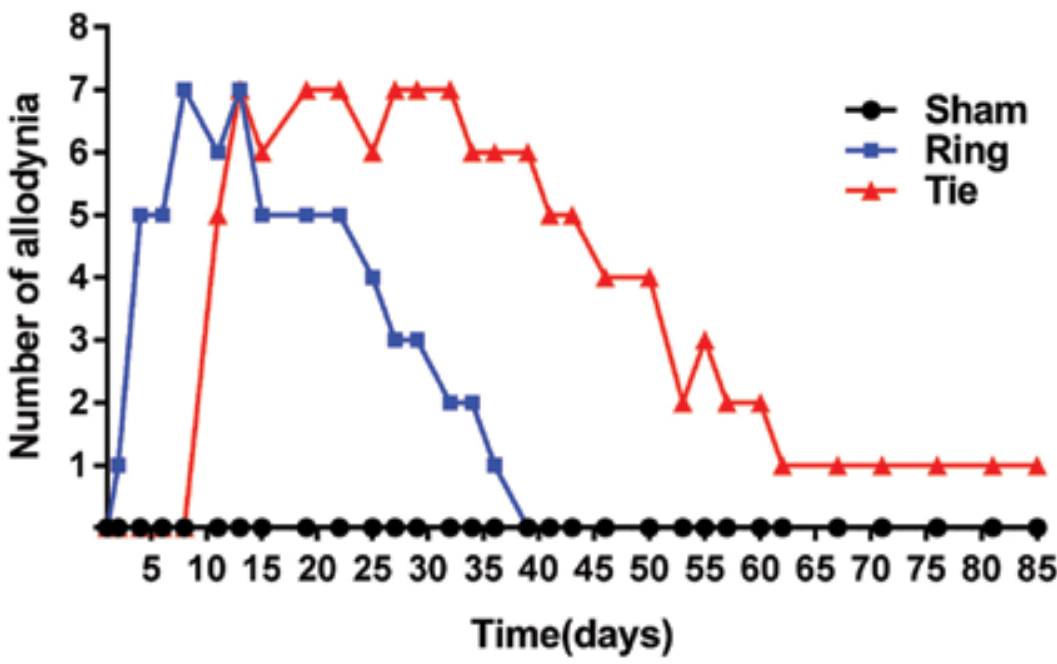

Fig. 8. Number of mice exhibiting allodynia (paw withdrawal threshold $<0.6)$ over time for each group

all time points until 12 weeks after reperfusion, when the research was terminated (Fig. 8).

\section{Histology}

The ring and tie groups exhibited significantly high intensity of TNF- $\alpha$ as compared with the sham group. There was no significant difference in TNF- $\alpha$ intensity between the ring and tie groups (Fig. 9).

\section{Discussion}

This study was conducted to design a new method for production of a CPIP mice model with higher levels of expression and longer duration of allodynia, which can be used in CRPS-I experiments, which is easier than existing methods.

The CPIP model has been used in numerous studies as an animal model for CRPS-I because mechanical allodynia and hyperalgesia following hyperemia and edema after reperfusion resemble the symptoms of CRPS-I in humans (5-7). We initially conducted an experiment in which medications were injected into a CPIP model with O-ring to reduce the pain caused by CRPS-I, in preparation for another experiment. Because the rate of successful production of the CPIP model is approximately $50 \%$ using O-rings in our laboratory, over twice as many animals were required for drug 


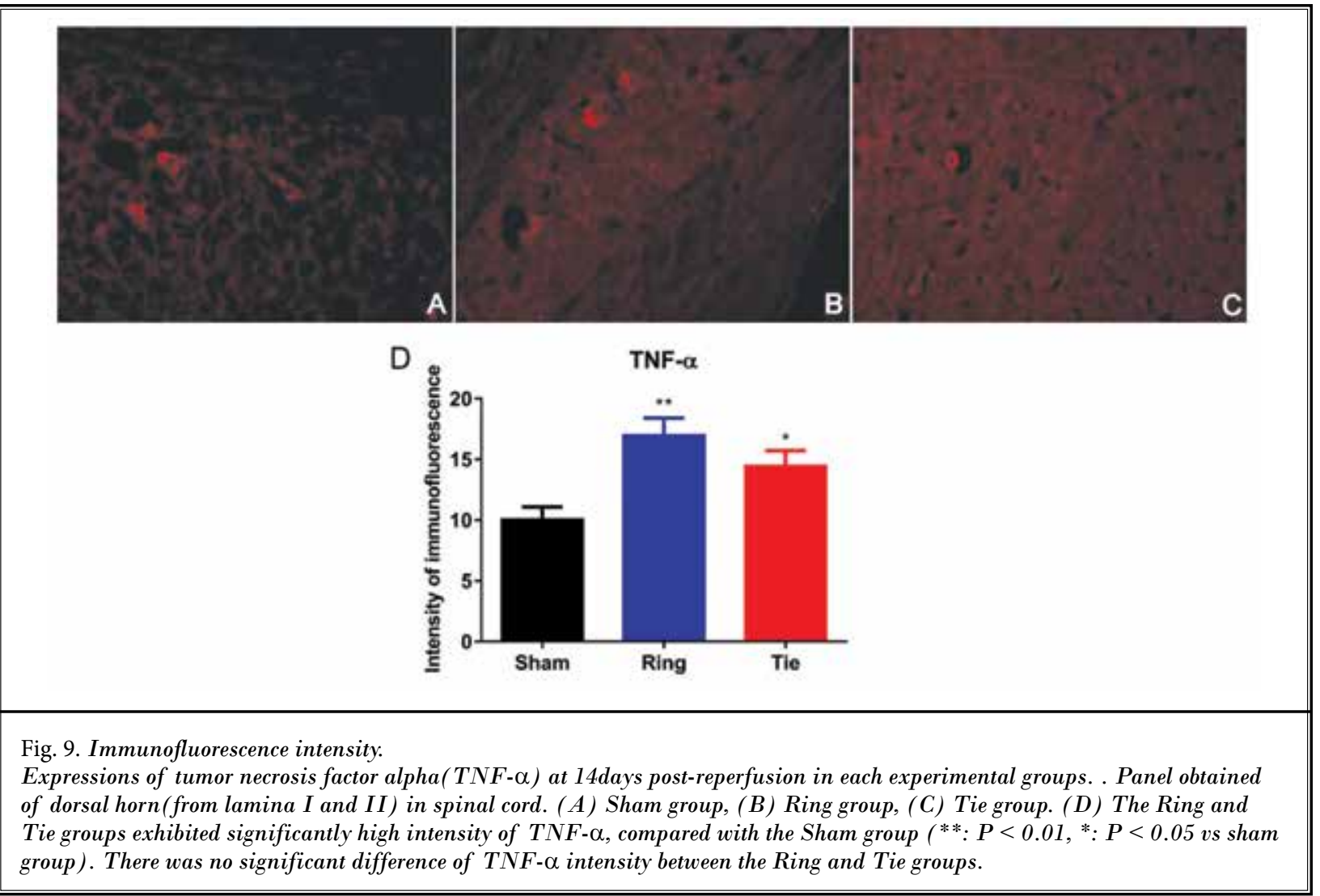

administration, and the overall research period became longer due to repeated model production. Moreover, because allodynia lasts approximately 1 month (2), it was difficult to observe the effect of long-term drug administration. Therefore we devised a method to produce a better model that could induce allodynia at a higher rate than the existing methods that use O-rings. An important factor during this process was to determine the tie force that would cause sufficient ischemia when the hind limb was tied, as well as to determine the appropriate number of times to tie the hind limb and the ischemia duration.

It is difficult to accurately measure the pressure applied to an animal limb in a CPIP model using an O-ring. In general, sufficient pressure must be applied to the blood vessels within a limb using a tourniquet to block arterial inflow but not so much as to damage the surrounding structures. Although pressures of $200-250 \mathrm{mmHg}$ and $250-350 \mathrm{mmHg}$ are applied to the upper and lower limbs in humans, respectively, using a tourniquet, how these values were determined has not been explained (8). Some surgeons believe that the pressure must be at least $70 \mathrm{mmHg}$ greater than the systolic pressure (9) or above $100-150 \mathrm{mmHg}$ to create a bloodless field (10). However, a modification of Bruner's 10 rules proposed in 1951 has been used as a guideline for determining the correct inflation pressure $(11,12)$. O-rings of size 7/32 ID (ISO sizing number 009) are usually used in CPIP model production in rats. It is impossible to know how much pressure these O-rings provide, and it appears that they have been empirically used. In this study, a CPIP model was produced using an O-ring with a 5/64-inch internal diameter (ISO sizing number 004) to fit the mice, but we could not measure the pressure applied to the mouse limb or determine whether the model effectively blocked blood flow to induce ischemia.

A push-pull gauge was used to tie a rubber band around the hind limb, once from the front with a force of $300 \mathrm{~g}$ and once again from the back with equal force. In our pilot study with 23 mice performed prior to this experiment, a tying force of $\geq 500 \mathrm{~g}$, repeated tying $\geq 3$ times, or maintaining ischemia for $\geq 5$ hours often led to necrosis in the hind paw. In the present study, which was conducted after a series of pilot studies, necrosis of the hind paw was not observed in a single mouse. 
Because the push-pull gauge can be easily adjusted, the tie point can be moved in the direction of the thigh with sufficient muscle to prevent necrosis of the bones (13).

We performed reperfusion 3 hours after inducing ischemia, as in the method of rat CPIP production used by Coderre et al (2). However, the duration of ischemia that is safe and does not cause necrosis is unclear because the tolerance of each tissue to ischemia varies among individuals. No clinical evidence of irreversible damages has been reported in human muscle until after 2 hours of ischemic time (14), moreover, no meaningful histological changes could be observed until after 3 hours of ischemic time in monkeys $(15,16)$. In a prior study, blood flow into the gracilis muscle of dogs was blocked for 3,4 , or 5 hours, and reperfusion was then induced for 48 hours; the rates of postischemic necrosis were evaluated: $2 \%, 30.5 \%$, and $90.1 \%$, respectively (17). Another study reported muscle necrosis after 4 hours of nutrient supply blocking in blood vessels (18). Therefore for the present study an ischemic time of 3 hours seemed appropriate. In cases of necrosis that occurred after 3 hours of ischemic time in the pilot study, it appears that the blood vessels were tied with an excessive amount of force, causing direct injury to tissues and subsequent necrosis.

CRPS-I usually occurs after fracture, sprain, contusion, crush injury, arthroscopic surgery, tight casting, or edematous soft tissue injury (19-21). A common characteristic of these injuries is that they induce an early inflammatory response and microvascular and ischemic changes in tissues. Although the exact pathophysiology of CRPS-I is unknown, a possible mechanism is posttraumatic inflammation $(22,23)$. Trauma gives rise to complex immune responses, induces the secretion of inflammatory cytokines, sensitizes the peripheral nociceptors to cause pain and hyperalgesia, and promotes neuropeptide secretion $(24,25)$. An inflammatory response induces microvascular permeability and plasma extravasation, and induces high arterial blood flow and peripheral venous pressure resulting in arteriovenous shunting, which contributes to the onset of ischemic contractures and compartment syndrome (26-29). In addition to inflammatory responses that continue after the initial injury, microcirculatory abnormalities that occur after ischemia-reperfusion could play an important role in the pathogenesis of CRPS-I $(2,19,20)$. Microcirculatory changes such as thrombosis, capillary endothelial cell swelling, and leukocyte plugging occur between 2 and 4 hours after reperfusion and increase vascular permeability to plasma proteins, leading to interstitial edema $(30,31)$. This edema can spread to muscles, joint capsules, and other anatomic structures as in compartment syndrome and eventually results in severe tissue edema. This series of events continuously induces tissue ischemia, sensitizes and activates afferent nociceptors in tissues, and causes central sensitization, which induces mechanical allodynia, hyperalgesia, and cold allodynia (32). In our experiment, the tie group showed more severe and longer lasting edema of the left hind limb than the ring group. Thus it appears that continuous inflammation and ischemic conditions induced central and peripheral sensitization in the tie group. As a result, the tie group had a higher incidence of mechanical allodynia than the ring group, with a duration of allodynia that extended to over 4 weeks. Based on these results, we found that the rate of successful CPIP animal model production and the duration of allodynia may increase as the severity of edema increases even if in humans edema is not always present and may be brief or alternatively.

Coderre et al (2) reported that mechanical allodynia occurs as early as 8 hours after reperfusion, reaches peak severity at 4 days, and is maintained for at least 4 weeks in $70 \%$ of rats in a CPIP model using Orings. In our experiment, however, mice began to have meaningful allodynia at 4 days after reperfusion in the ring group when a CPIP model using existing O-rings was used; moreover, allodynia was maintained at peak severity from 1 to 2 weeks after reperfusion, and the severity gradually decreased. At 4 weeks after reperfusion, only 2 of $8(25 \%)$ mice showed allodynia, with later onset and shorter duration than in rats. The tie group had a more delayed onset of allodynia than the ring group. The tie group showed a reduced response to von Frey filaments compared with the sham and ring groups. The reason for this could be because the tie group showed severe edema until 6 days after reperfusion, therefore tactile sensation decreased during this period. Mechanical allodynia gradually increased as the edema reduced in the tie group. Similar observations have been made in numerous pilot studies. In the tie group, 7 of $8(87.5 \%)$ mice showed mechanical allodynia starting at 13 days after reperfusion. Mechanical allodynia was maintained until 4 weeks after reperfusion, and over $50 \%$ of the mice showed mechanical allodynia until 6 weeks. At 12 weeks, which is when the research terminated, one mouse had a von Frey threshold below 0.2. In a pilot study performed before this experiment, the duration of allodynia was 0.03 in terms of the von 
Frey response in the tie group, and allodynia was maintained in 2 of 9 mice for 72 days. The average duration of allodynia was 30 days longer in the tie group than in the ring group.

Symptoms spread to the contralateral side in about $16 \%$ of all patients with CRPS-I $(19,33)$, and there have been reports of symptom contraction in contralateral unlesioned structures following peripheral nerve damage in animal experiments (34). The contralateral effects are usually mild and brief. In our experiment, allodynia sporadically occurred in the contralateral hind paw at 22 and 50 days and was more significant in the tie group than the sham group. Whether this phenomenon was incidental or had a biological purpose is unclear. However, the central mechanism, in which signaling through the commissural interneuron system of the spinal cord and brain stem that allow both sides of the body to be connected, is currently the best explanation. It appears that whether or not contralateral effects occur depends on the severity of central sensitization after a muscle or tissue injury (35), and the contralateral effects observed in our experiment seem to indicate a higher severity of central sensitization in the tie group than in the ring group.

TNF- $\alpha$ is generally considered a proinflammatory cytokine and is related to the pathology of CRPS (3639). Huygen et al (37) found that TNF- $\alpha$ levels were significantly increased in the affected arm of patients versus the unaffected arm. In the rat sciatic nerve that underwent ischemia/reperfusion injury, expression levels of TNF- $\alpha$ increased at 6 to 12 hours postreperfusion and had remained 2-fold higher at 7 days postreperfusion, as compared with baseline levels (38). We also confirmed that expressions of TNF- $\alpha$ in the ring and tie groups were significantly increased than in the sham group at 14 days postreperfusion. We sacrificed the mice at 14 days postreperfusion because of this in the pilot experiment and present study, the tie group showed prominent mechanical allodynia after 10 days postreperfusion. There was no difference in the expression of TNF- $\alpha$ between the ring and tie groups, these results are consistent with the reported data that the level of TNF- $\alpha$ does not correlate with severity of disease or pain intensity $(36,39)$.

This study has limitations. First, we did not evaluate hyperalgesia or cold and heat allodynia. Second, we did not measure blood levels of inflammatory or antiinflammatory cytokines, or assess oxidative stress biomarkers such as isoprostane, 8-hydroxy-2'-deoxyguanosine (a marker of DNA oxidative damage), and malondialdehyde.

Future research should examine prolonged hind limb ischemia-reperfusion, which is known to induce inflammatory cascades and contribute to the formation of reactive oxygen species $(1,7,40-43)$.

\section{Conclusions}

Our CPIP model that uses a new tying technique can apply uniform pressure regardless of body weight and size using rubber bands of similar elasticity, and therefore has a higher success rate of model production compared with the use of O-rings. We believe that if the elasticity coefficient $\left(0.588 \mathrm{kgf} / \mathrm{cm}^{2}\right)$ and tie force $(300$ g) of the rubber band are similar, then the CPIP animal modeling is likely to be reproducible. The model can shorten the overall research time, reduce the number of animals sacrificed in an experiment, and lengthen the duration of allodynia, allowing follow-up of drug effects over 1 month; thus the model is anticipated to be useful in CRPS-I research.

\section{RefEREnCES}

1. Bussa M, Guttilla D, Lucia M, Mascaro A, Rinaldi S. Complex regional pain syndrome type I: A comprehensive review. Acta Anaesthesiol Scand 2015; 59:685-697.

2. Coderre TJ, Xanthos DN, Francis L, Bennett GJ. Chronic post-ischemia pain (CPIP): A novel animal model of complex regional pain syndrome-type I (CRPS-I; reflex sympathetic dystrophy) produced by prolonged hindpaw ischemia and reperfusion in the rat. Pain
2004; 112:94-105.

3. Tang C, Li J, Tai WL, et al. Sex differences in complex regional pain syndrome type I (CRPS-I) in mice. J Pain Res 2017; 10:1811-1819.

4. Bonin RP, Bories C, De Koninck Y. A simplified up-down method (SUDO) for measuring mechanical nociception in rodents using von Frey filaments. Mol Pain 2014; 10:26.
5. Birklein F, Riedl B, Sieweke N, Weber $M$, Neundorfer B. Neurological findings in complex regional pain syndromes-analysis of 145 cases. Acta Neurol Scand 2000; 101:262-269.

6. Wasner G, Schattschneider J, Heckmann K, Maier C, Baron R. Vascular abnormalities in reflex sympathetic dystrophy (CRPS I): Mechanisms and diagnostic value. Brain 2001; 124:587-599. 
7. Ryu TH, Jung KY, Ha MJ, Kwak KH, Lim DG, Hong JG. Superoxide and nitric oxide involvement in enhancing of $\mathrm{N}$ methyl-D-aspartate receptor-mediated central sensitization in the chronic post-ischemia pain model. Korean J Pain 2010; 23:1-10.

8. Azar FM, Canale ST, Beaty JH. Campbell's Operative Orthopaedics E-Book. Amsterdam, Elsevier Health Sciences, 2016.

9. Sanders R. The tourniquet. Instrument or weapon? Hand 1973; 5:119-123.

10. Rorabeck $\mathrm{CH}$. Tourniquet-induced nerve ischemia: An experimental investigation. J Trauma 1980; 20:280-286.

11. Kutty S, McElwain JP. Padding under tourniquets in tourniquet controlled surgery: Bruner's ten rules revisited. Injury 2002; 33:75.

12. Bruner JM. Safety factors in the use of the pneumatic tourniquet for hemostasis in surgery of the hand. J Bone Joint Surg Am 1951; 33A:221-224.

13. Fletcher IR, Healy TE. The arterial tourniquet. Ann R Coll Surg Engl 1983; 65:409-417.

14. Solonen KA, Hjelt L. Morphological changes in striated muscle during ischaemia. A clinical and histological study in man. Acta Orthop Scand 1968; 39:13-19.

15. Tountas CP, Bergman RA. Tourniquet ischemia: Ultrastructural and histochemical observations of ischemic human muscle and of monkey muscle and nerve. J Hand Surg Am 1977; 2:31-37.

16. Patterson S, Klenerman L. The effect of pneumatic tourniquets on the ultrastructure of skeletal muscle. J Bone Joint Surg $\mathrm{Br}$ 1979; 61-B:178-183.

17. Labbe R, Lindsay T, Walker PM. The extent and distribution of skeletal muscle necrosis after graded periods of complete ischemia. J Vasc Surg 1987; 6:152-157.

18. Makitie J, Teravainen H. Histochemical studies of striated muscle after temporary ischemia in the rat. Acta Neuropathol 1977; 37:101-109.

19. Allen G, Galer BS, Schwartz L. Epidemiology of complex regional pain syndrome: A retrospective chart review of 134 patients. Pain 1999; 80:539-544.

20. Galer BS, Henderson J, Perander J, Jensen MP. Course of symptoms and qual- ity of life measurement in complex regional pain syndrome: A pilot survey. J Pain Symptom Manage 2000; 20:286-292.

21. Sandroni P, Benrud-Larson LM, McClelland RL, Low PA. Complex regional pain syndrome type I: Incidence and prevalence in Olmsted county, a populationbased study. Pain 2003; 103:199-207.

22. Birklein F, Dimova V. Complex regional pain syndrome-up-to-date. Pain Rep 2017; 2:e624.

23. Goris RJ. Reflex sympathetic dystrophy: Model of a severe regional inflammatory response syndrome. World J Surg 1998; 22:197-202.

24. Birklein F, Drummond PD, Li W, et al. Activation of cutaneous immune responses in complex regional pain syndrome. J Pain 2014; 15:485-495.

25. Opree A, Kress M. Involvement of the proinflammatory cytokines tumor necrosis factor-alpha, IL-1 beta, and IL-6 but not IL-8 in the development of heat hyperalgesia: Effects on heat-evoked calcitonin gene-related peptide release from rat skin. J Neurosci 2000; 20:6289-6293.

26. Hoover TJ, Siefert JA. Soft tissue complications of orthopedic emergencies. Emerg Med Clin North Am 2000; 18:115139, vi.

27. Matsumura $\mathrm{H}$, Jimbo $\mathrm{Y}$, Watanabe $\mathrm{K}$. Haemodynamic changes in early phase reflex sympathetic dystrophy. Scand J Plast Reconstr Surg Hand Surg 1996; 30:133-138.

28. Oyen WJ, Arntz IE, Claessens RM, Van der Meer JW, Corstens FH, Goris RJ. Reflex sympathetic dystrophy of the hand: An excessive inflammatory response? Pain 1993; 55:151-157.

29. Schurmann M, Zaspel J, Gradl G, Wipfel A, Christ F. Assessment of the peripheral microcirculation using computerassisted venous congestion plethysmography in post-traumatic complex regional pain syndrome type I. J Vasc Res 2001; 38:453-461.

30. Harris AG, Steinbauer M, Leiderer R, Messmer K. Role of leukocyte plugging and edema in skeletal muscle ischemiareperfusion injury. Am J Physiol 1997; 273: $\mathrm{H} 989-996$.

31. Strock PE, Majno G. Microvascular changes in acutely ischemic rat muscle.
Surg Gynecol Obstet 1969; 129:1213-1224.

32. Wall PD, Woolf CJ. Muscle but not cutaneous C-afferent input produces prolonged increases in the excitability of the flexion reflex in the rat. J Physiol 1984; 356:443-458.

33. Maleki J, LeBel AA, Bennett GJ, Schwartzman RJ. Patterns of spread in complex regional pain syndrome, type I (reflex sympathetic dystrophy). Pain 2000; 88:259-266.

34. Koltzenburg M, Wall PD, McMahon SB. Does the right side know what the left is doing? Trends Neurosci 1999; 22:122-127.

35. Woolf CJ, Wall PD. Relative effectiveness of $C$ primary afferent fibers of different origins in evoking a prolonged facilitation of the flexor reflex in the rat. J Neurosci 1986; 6:1433-1442.

36. Hauser J, Hsu B, Nader ND. Inflammatory processes in complex regional pain syndromes. Immunol Invest 2013; 42:263-272.

37. Huygen FJ, De Bruijn AG, De Bruin MT, Groeneweg JG, Klein J, Zijlstra FJ. Evidence for local inflammation in complex regional pain syndrome type 1. Mediators Inflamm 2002; 11:47-51.

38. Ke T, Li R, Chen W. Inhibition of the NMDA receptor protects the rat sciatic nerve against ischemia/reperfusion injury. Exp Ther Med 2016; 11:1563-1572.

39. Dirckx M, Stronks DL, van BodegravenHof EA, Wesseldijk F, Groeneweg JG, Huygen FJ. Inflammation in cold complex regional pain syndrome. Acta Anaesthesiol Scand 2015; 59:733-739.

40. Blaisdell FW. The pathophysiology of skeletal muscle ischemia and the reperfusion syndrome: A review. Cardiovasc Surg 2002; 10:620-630.

41. Yassin MM, Barros D'Sa AA, Parks TG, et al. Lower limb ischaemia-reperfusion injury alters gastrointestinal structure and function. Br J Surg 1997; 84:1425-1429.

42. Kwak KH, Han CG, Lee SH, et al. Reactive oxygen species in rats with chronic post-ischemia pain. Acta Anaesthesiol Scand 2009; 53:648-656.

43. Han CG, Han JK, Park KB, Kwak KH, Park SS, Lim DG. Effect of superoxide on the development and maintenance of mechanical allodynia in a rat model of chronic post-ischemia pain. Korean J Anesthesiol 2012; 63:149-156. 